\title{
PAT-Facilitated Pharmaceutical Crystallization Development through Mechanistic Understanding
}

Peter Fung ${ }^{1}$, Helen Mah', Alexander F. G. Goldberg' ${ }^{1}$ Curtis Rieder' ${ }^{2}$ Ho-Yan Sun ${ }^{2}$,Zhuoyi Su²,

Juana Dü ${ }^{1 *}$, Chiajen Lai ${ }^{1 *}$

${ }^{1}$ Gilead Sciences, Inc., Foster City, CA 94404, USA

${ }^{2}$ Gilead Alberta ULC, Edmonton, Alberta T6S 1A1, CANADA 


\section{Supporting Information}

SI.1 Quantitative X-ray Analysis

The X-ray patterns of Compound X freebase and API (Maleate) have distinct features in the 5-15 ${ }^{\circ} 2 \theta$ region, as shown in Figure SI.1.1. Standard reference samples of physical mixtures of Compound X freebase and Maleate were prepared in 10\% increments from pure freebase to pure Maleate, as shown in Table SI.1.1 below. These samples were subjected to1-hr scan in X-ray, and characteristic peaks were integrated as shown in Figure SI.1.2 to Figure SI.1.4.

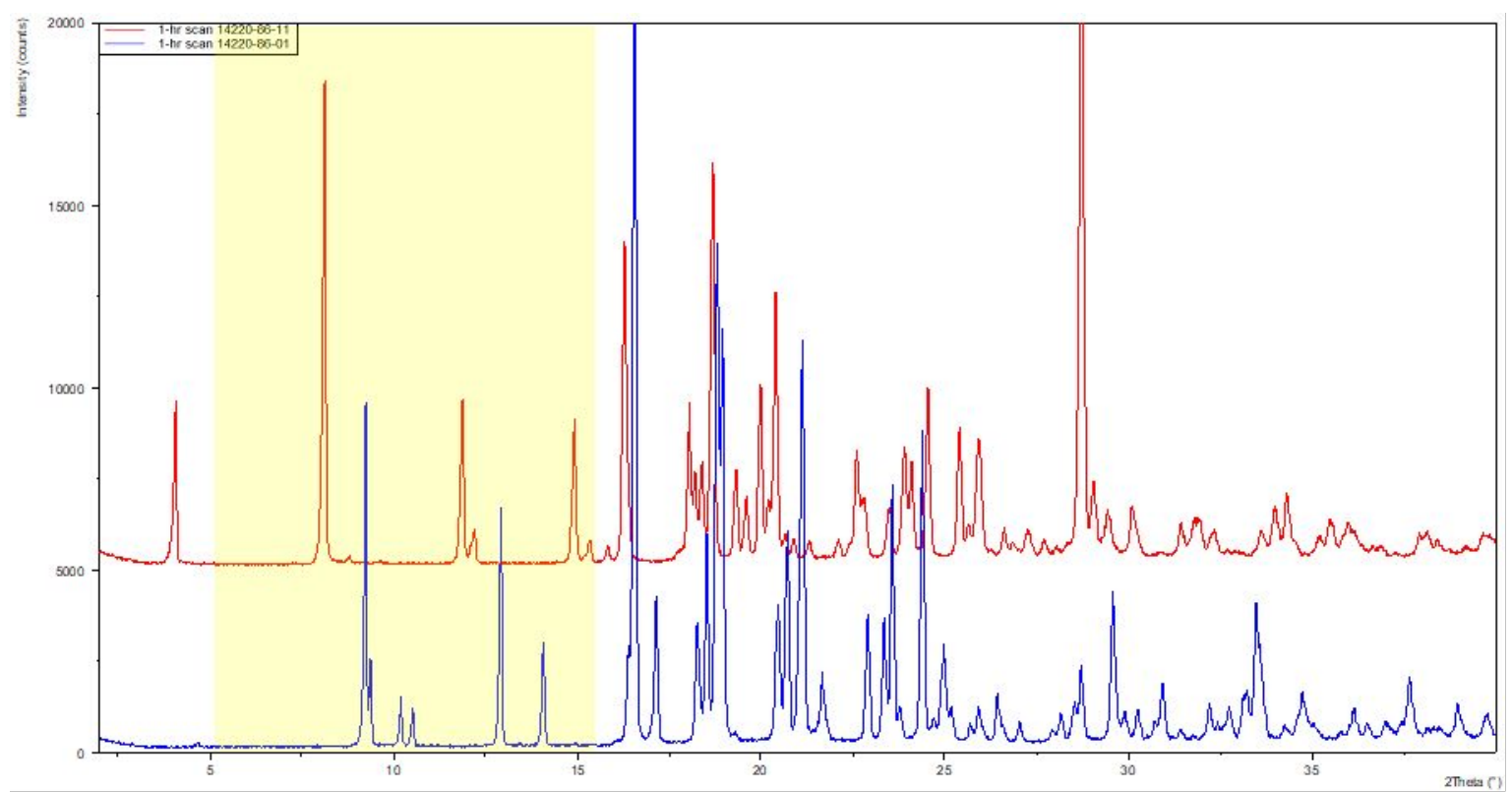

Figure SI1.1. X-ray patterns of Compound X freebase (bottom, blue curve) and Compound X API (Maleate, top red curve). X-ray peaks at 12.9 and $8.1^{\circ} 2 \theta$ in the highlighted region were used for quantitation. 
Table SI1.1. Peak area analysis of calibration samples

\begin{tabular}{|c|c|c|c|c|c|c|}
\hline Sample & $\begin{array}{c}\text { Freebase } \\
(\mathrm{mg})\end{array}$ & $\begin{array}{c}\text { Maleate } \\
(\mathrm{mg})\end{array}$ & $\begin{array}{c}\mathrm{wt} \% \\
\text { Freebase }\end{array}$ & $\begin{array}{c}\text { Peak area } \\
@ 12.9^{\circ}\end{array}$ & $\begin{array}{c}\text { Peak area } \\
@ 8.1^{\circ}\end{array}$ & $\begin{array}{c}\text { Peak @ } \\
12.9^{\circ} / \text { peak @ } \\
12.9^{\circ}+\text { peak @ } \\
\left.8.1^{\circ}\right)\end{array}$ \\
\hline Calibration 1 & 2000 & 0 & 1 & 636.8 & 0 & 1 \\
\hline Calibration 2 & 1800 & 200 & 0.9 & 670.6 & 53.6 & 0.926 \\
\hline Calibration 3 & 1600 & 400 & 0.8 & 590.1 & 109 & 0.844 \\
\hline Calibration 4 & 1400 & 600 & 0.7 & 522.1 & 233.9 & 0.691 \\
\hline Calibration 5 & 1200 & 800 & 0.6 & 472 & 316.7 & 0.598 \\
\hline Calibration 6 & 1000 & 1000 & 0.5 & 391.7 & 346.2 & 0.531 \\
\hline Calibration 7 & 800 & 1200 & 0.4 & 346.8 & 684.6 & 0.336 \\
\hline Calibration 8 & 600 & 1400 & 0.3 & 271.9 & 746.4 & 0.267 \\
\hline Calibration 9 & 400 & 1600 & 0.2 & 187.6 & 1011 & 0.157 \\
\hline Calibration 10 & 200 & 1800 & 0.1 & 121.1 & 1112.3 & 0.098 \\
\hline Calibration 11 & 0 & 2000 & 0 & 0 & 1398.9 & 0 \\
\hline
\end{tabular}



Figure SI.1.2. Calibration sample 1: 100\% freebase and 0\% Maleate. Peak characteristic of freebase at $\sim 12.9^{\circ} 2 \theta$ is integrated as shown. 


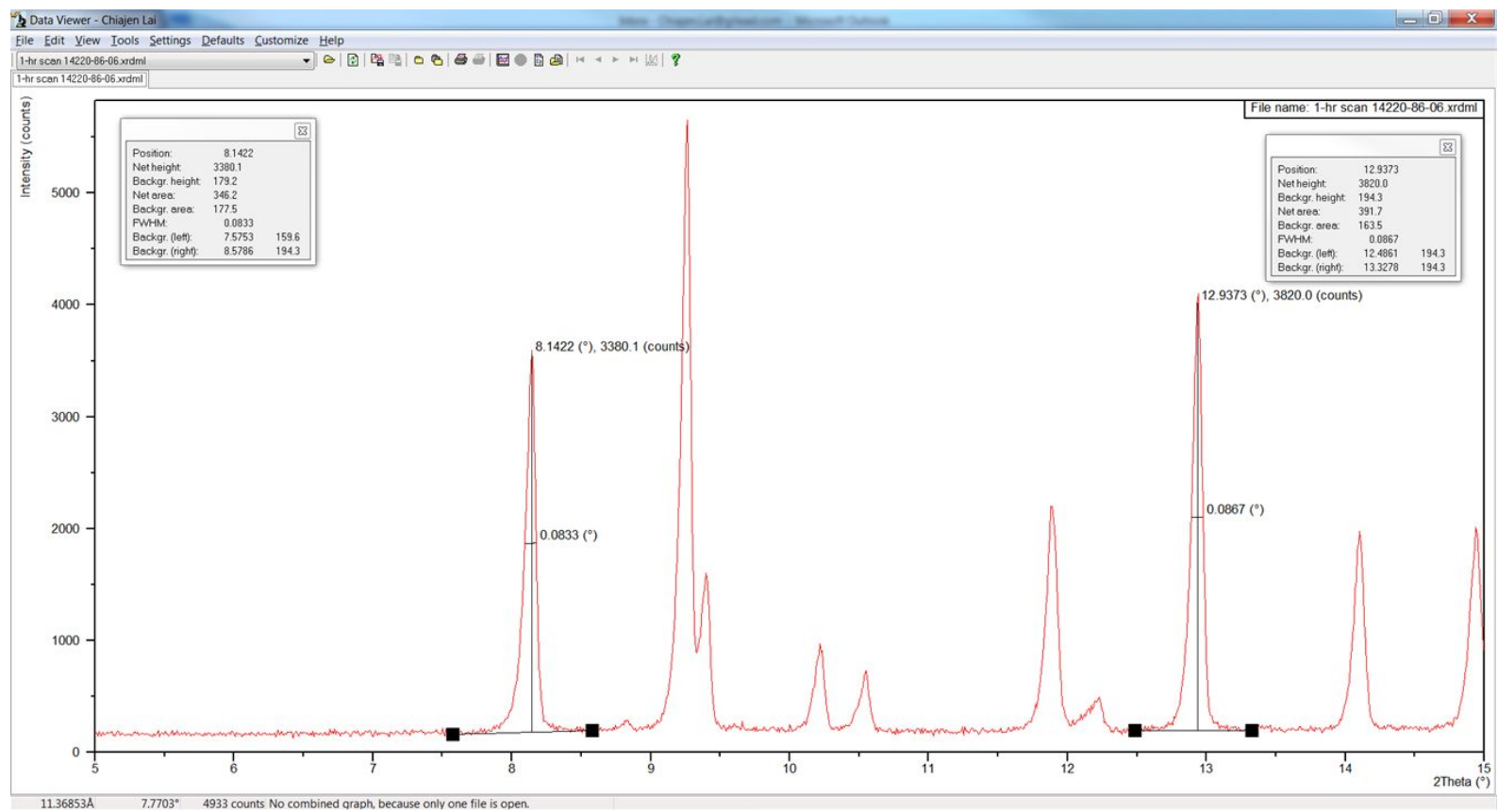

Figure SI.1.3. Calibration sample 6 (as example): 50\% freebase and 50\% Maleate. Peaks characteristic of freebase at $\sim 12.9^{\circ} 2 \theta$ and Maleate at $\sim 8.1^{\circ} 2 \theta$ are integrated as shown.

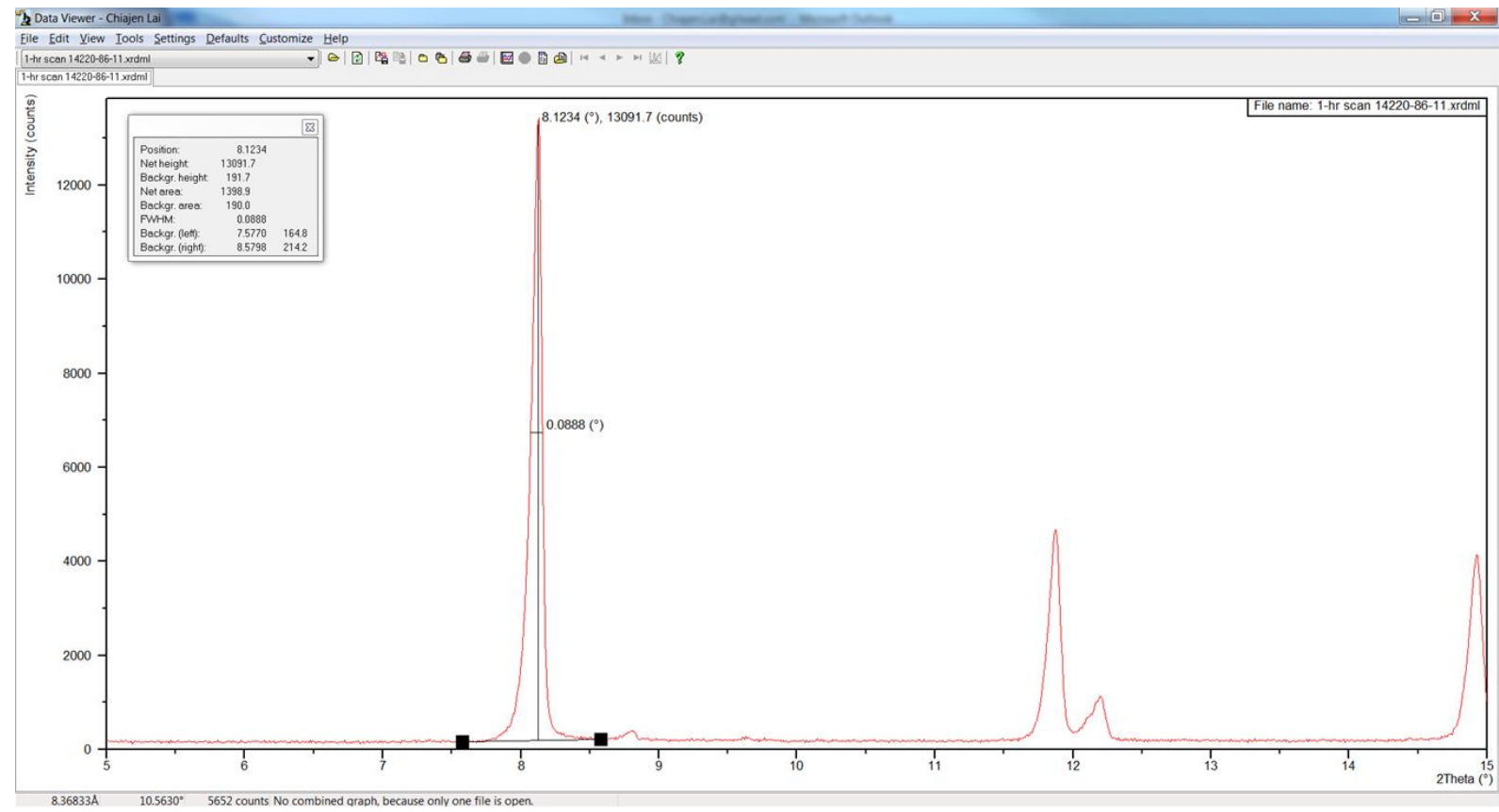

Figure SI.1.4. Calibration sample 11: 0\% freebase and 100\% Maleate. Peak characteristic of Maleate at $\sim 8.1^{\circ} 2 \theta$ is integrated as shown. 
Figure SI.1.5 shows the calibration curve based on the peak ratios of the samples. Determination of percent conversion in the process samples was achieved by running the samples at the same condition (1-hr scan) to utilize the calibration curve. The values are reported in Table 2.

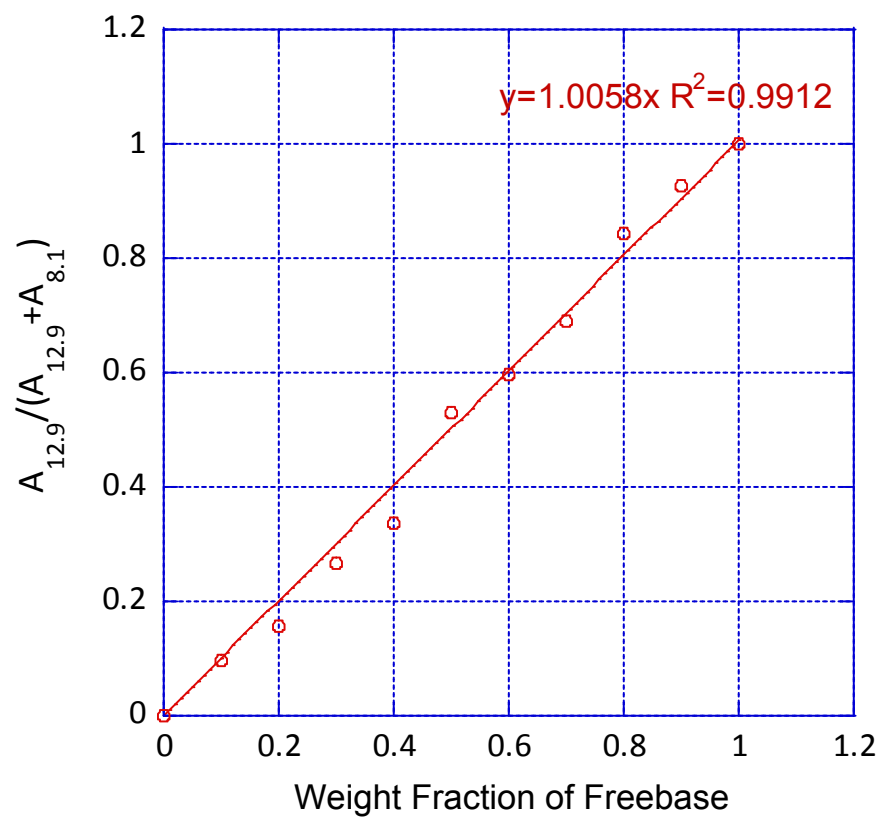

Figure SI.1.5. X-ray calibration curve of freebase/Maleate mixtures 
SI.2 Crystallization process conditions and API characterization by particle size (Malvern and FBRM).

\begin{tabular}{|c|c|c|c|c|c|c|c|c|c|c|c|c|c|c|}
\hline \multirow[b]{2}{*}{ Exp \# } & \multirow[b]{2}{*}{ Lot } & \multirow[b]{2}{*}{$\begin{array}{l}\text { Acetone/ } \\
\mathrm{H} 2 \mathrm{O} \mathrm{v} / \mathrm{v}\end{array}$} & \multirow[b]{2}{*}{$\begin{array}{l}\text { Agitation } \\
(\mathrm{rpm})\end{array}$} & \multirow[b]{2}{*}{$\begin{array}{l}\text { Freebase } \\
\text { form }\end{array}$} & \multirow[b]{2}{*}{ Seeds } & \multirow[b]{2}{*}{ Temp $\left({ }^{\circ} \mathrm{C}\right)$} & \multirow[b]{2}{*}{$\begin{array}{l}\text { Acid add. } \\
\text { (hr) }\end{array}$} & \multicolumn{5}{|c|}{ Malvern Mastersizer 3000} & \multicolumn{2}{|l|}{ FRBM } \\
\hline & & & & & & & & $\mathrm{D} 10$ & D50 & D90 & $\mathrm{D}[4,3]$ & Span & MSW & $90 \% \mathrm{SW}$ \\
\hline 1 & M-26 & $50 / 50$ & 500 & $\mathrm{I}$ & None & 50 & 0 & 28.7 & 92.5 & 170 & 96.1 & 1.524 & 168.8 & 300.8 \\
\hline 2 & M-28 & $50 / 50$ & 500 & $\mathrm{I}$ & Large & 50 & 0 & 25.6 & 97.5 & 232 & 115 & 2.121 & 169.8 & 304.5 \\
\hline 3 & M-29 & $50 / 50$ & 500 & $\mathrm{I}$ & None & 50 & 0 & 30.9 & 104 & 207 & 112 & 1.693 & 175.7 & 311.5 \\
\hline 4 & M-30 & $50 / 50$ & 500 & I & None & 40 & 0 & 15.2 & 68 & 150 & 75.6 & 1.980 & 126.9 & 232.8 \\
\hline 5 & M-31 & $50 / 50$ & 500 & I & None & 30 & 0 & 7.4 & 36.8 & 111 & 49.4 & 2.809 & 87.6 & 170.9 \\
\hline 6 & M-32 & $50 / 50$ & 500 & $\mathrm{I}$ & None & 20 & 0 & 3.3 & 16 & 54.1 & 22.8 & 3.180 & 81.7 & 159.2 \\
\hline 7 & M-33 & $50 / 50$ & 500 & I & Large & 20 & 0 & 3.6 & 19.8 & 79.4 & 31.8 & 3.836 & 80.4 & 156.2 \\
\hline 8 & M-34 & $60 / 40$ & 500 & $\mathrm{I}$ & None & 50 & 0 & 28.1 & 111 & 219 & 118 & 1.717 & 179.5 & 318.8 \\
\hline 9 & M-35 & $70 / 30$ & 500 & $\mathrm{I}$ & None & 50 & 0 & 32.9 & 102 & 250 & 126 & 2.136 & 155.0 & 273.9 \\
\hline 10 & M-36 & $80 / 20$ & 500 & $\mathrm{I}$ & None & 50 & 0 & 24.6 & 89.4 & 220 & 107 & 2.180 & 135.8 & 241.9 \\
\hline 11 & M-37 & $90 / 10$ & 500 & I & None & 50 & 0 & 15.4 & 63.8 & 139 & 70.8 & 1.935 & 104.8 & 197.8 \\
\hline 12 & M-38 & $95 / 5$ & 500 & I & None & 50 & 0 & 6.9 & 34.5 & 113 & 48.4 & 3.067 & 67.5 & 136.9 \\
\hline 13 & M-39 & $95 / 5$ & 500 & I & Large & 50 & 0 & 7.3 & 37.2 & 135 & 55.9 & 3.429 & 62.9 & 128.8 \\
\hline 14 & M-40 & $95 / 5$ & 500 & $\mathrm{I}$ & None & 50 & 0 & 7.6 & 33.1 & 93.3 & 42.7 & 2.589 & 63.2 & 125.3 \\
\hline 15 & M-41 & $95 / 5$ & 500 & I & None & 40 & 0 & 4.9 & 23 & 80 & 33.5 & 3.266 & 62.6 & 130.7 \\
\hline 16 & M-43 & $95 / 5$ & 500 & I & None & 30 & 0 & 3.2 & 15.3 & 50.9 & 21.7 & 3.121 & 55.1 & 117.6 \\
\hline 17 & M-44 & $95 / 5$ & 500 & I & None & 20 & 0 & 2 & 8.3 & 28.9 & 14.5 & 3.257 & 48.9 & 108.8 \\
\hline 18 & M-45 & $95 / 5$ & 500 & I & Large & 20 & 0 & 2.3 & 10.6 & 37 & 16.5 & 3.282 & 43.6 & 93.5 \\
\hline 19 & M-46-02 & $95 / 5$ & 500 & $\mathrm{I}$ & None & 20 & 0 & 2.4 & 10.3 & 34.3 & 14.8 & 3.113 & 44.3 & 92.7 \\
\hline 20 & M-46-03 & $95 / 5$ & 500 & $\mathrm{I}$ & None & 50 & 0 & 2.6 & 10.6 & 30.8 & 14 & 2.658 & 77.6 & 158.3 \\
\hline 21 & M-47 & $95 / 5$ & 500 & $\mathrm{I}$ & None & 50 & 1 & 7.8 & 32.4 & 96.9 & 43.6 & 2.752 & 71.4 & 142.3 \\
\hline 22 & M-48-02 & $50 / 50$ & 500 & I & None & 20 & 0 & 2.7 & 14.9 & 54.0 & 22.1 & 3.455 & 80.1 & 157.3 \\
\hline 23 & M-48-03 & $50 / 50$ & 500 & $\mathrm{I}$ & None & 50 & 0 & 3.9 & 17.7 & 46 & 22 & 2.376 & 80.1 & 159.2 \\
\hline 24 & M-49 & $95 / 5$ & 500 & $\mathrm{I}$ & None & 50 & 2 & 9.7 & 37.3 & 101 & 47.7 & 2.452 & 38.2 & 75.7 \\
\hline 25 & M-50 & $95 / 5$ & 500 & $\mathrm{I}$ & None & $20->50$ & 0 & 2.3 & 14 & 48.4 & 20.4 & 3.283 & 65.2 & 133.7 \\
\hline 26 & M-51 & $95 / 5$ & 500 & $\mathrm{I}$ & None & 50 & 3 & 11.9 & 42.8 & 107 & 52.3 & 2.231 & 42.7 & 83.9 \\
\hline 27 & M-52 & $95 / 5$ & 500 & $\mathrm{I}$ & None & $20->50$ & 0 & 5.8 & 29.2 & 82 & 37.4 & 2.607 & 69.2 & 141.1 \\
\hline 28 & $\mathrm{~N}-04$ & $100 / 0$ & 500 & $\mathrm{I}$ & None & 20 & 0 & 1.1 & 4 & 17.9 & 6.9 & 4.193 & 24.8 & 40.8 \\
\hline 29 & $\mathrm{~N}-05$ & $100 / 0$ & 500 & $\mathrm{I}$ & None & 20 & 0 & 1.5 & 6.1 & 16.1 & 7.6 & 2.405 & 24.1 & 39.9 \\
\hline 30 & $\mathrm{~N}-06$ & $95 / 5$ & 500 & I & None & 20 & 0 & 2.3 & 9.5 & 32.4 & 13.8 & 3.169 & 22.9 & 38.2 \\
\hline 31 & N-07 & $90 / 10$ & 500 & I & None & 20 & 0 & 4.1 & 18.4 & 67.7 & 27.7 & 3.464 & 27.2 & 44.4 \\
\hline
\end{tabular}




\begin{tabular}{|c|c|c|c|c|c|c|c|c|c|c|c|c|c|c|}
\hline 32 & $\mathrm{~N}-08$ & $80 / 20$ & 500 & II & None & 20 & 0 & 6.5 & 29.8 & 99.4 & 42.5 & 3.116 & 31.1 & 54.2 \\
\hline 33 & $\mathrm{~N}-09$ & $70 / 30$ & 500 & $\mathrm{I}$ & None & 20 & 0 & 6.2 & 33.6 & 106 & 46.1 & 2.962 & 30.4 & 52.6 \\
\hline 34 & $\mathrm{~N}-10$ & $60 / 40$ & 500 & $\mathrm{I}$ & None & 20 & 0 & 3.7 & 20.7 & 63.1 & 27.7 & 2.864 & 34.3 & 67.0 \\
\hline 35 & N-11 & $50 / 50$ & 500 & $\mathrm{I}$ & None & 20 & 0 & 1.8 & 8.4 & 49.3 & 17.6 & 5.685 & 28.2 & 51.0 \\
\hline 36 & N-12 & $95 / 5$ & 800 & $\mathrm{I}$ & None & 20 & 0 & 1.9 & 7.1 & 26.6 & 11.1 & 3.489 & 27.7 & 42.3 \\
\hline 37 & N-13 & $95 / 5$ & 300 & $\mathrm{I}$ & None & 20 & 0 & 3 & 16.2 & 59.3 & 24.3 & 3.473 & 26.7 & 44.3 \\
\hline 38 & N-14 & $95 / 5$ & 500 & $\mathrm{I}$ & None & 20 & 0 & 1.8 & 8.3 & 33.2 & 13.4 & 3.772 & 24.9 & 42.1 \\
\hline 39 & $\mathrm{~N}-15$ & $95 / 5$ & 500 & II & None & 20 & 0 & 1.9 & 8.6 & 48.6 & 18.1 & 5.422 & 38.5 & 67.1 \\
\hline 40 & N-16 & $95 / 5$ & 500 & $\mathrm{I}$ & None & 20 & 0 & 2.2 & 10 & 37 & 15.3 & 3.478 & 23.8 & 40.0 \\
\hline 41 & N-17 & $95 / 5$ & 500 & $\mathrm{I}$ & None & 20 & 0 & 1.8 & 8.8 & 32.6 & 13.4 & 3.497 & 25.7 & 43.8 \\
\hline 42 & N-19 & $100 / 0$ & 500 & $\mathrm{I}$ & None & 50 & 0 & 2.0 & 8.3 & 27.2 & 11.9 & 3.027 & 22.4 & 36.5 \\
\hline 43 & $\mathrm{~N}-20$ & $60 / 40$ & 500 & $\mathrm{I}$ & None & 30 & 0 & 10.7 & 49.8 & 114 & 56.6 & 2.071 & 47.4 & 83.6 \\
\hline 44 & N-21 & $70 / 30$ & 500 & $\mathrm{I}$ & None & 30 & 0 & 14.6 & 58.7 & 131 & 66.3 & 1.986 & 48.9 & 85.5 \\
\hline 45 & $\mathrm{~N}-22$ & $100 / 0$ & 500 & $\mathrm{I}$ & None & 30 & 0 & 1.4 & 5.5 & 26.6 & 9.9 & 4.563 & 24.5 & 41.1 \\
\hline 46 & $\mathrm{~N}-23$ & $80 / 20$ & 500 & $\mathrm{I}$ & None & 30 & 0 & 12.2 & 52.5 & 119 & 59.4 & 2.025 & 43.2 & 75.9 \\
\hline 47 & $\mathrm{~N}-24$ & $90 / 10$ & 500 & $\mathrm{I}$ & None & 30 & 0 & 7.9 & 33.9 & 89.5 & 42.3 & 2.410 & 32.0 & 54.5 \\
\hline 48 & $\mathrm{~N}-25$ & $60 / 40$ & 500 & $\mathrm{I}$ & None & 40 & 0 & 22.8 & 83.0 & 157 & 86.2 & 1.612 & 68.2 & 114.6 \\
\hline 49 & $\mathrm{~N}-26$ & $70 / 30$ & 500 & $\mathrm{I}$ & None & 40 & 0 & 23.4 & 86.9 & 182 & 95.1 & 1.825 & 67.7 & 114.3 \\
\hline 50 & $\mathrm{~N}-27$ & $80 / 20$ & 500 & $\mathrm{I}$ & None & 40 & 0 & 20.5 & 78.0 & 161 & 84.5 & 1.798 & 62.6 & 106.9 \\
\hline 51 & N-28 & $90 / 10$ & 500 & $\mathrm{I}$ & None & 40 & 0 & 10.1 & 39.3 & 108 & 50.3 & 2.483 & 43.1 & 77.1 \\
\hline 52 & $\mathrm{~N}-29$ & $100 / 0$ & 500 & $\mathrm{I}$ & None & 40 & 0 & 2.0 & 6.9 & 23.3 & 10.1 & 3.104 & 19.2 & 33.4 \\
\hline 53 & $\mathrm{~N}-30(2.5 \mathrm{eq})$ & $95 / 5$ & 500 & $\mathrm{I}$ & None & 20 & 0 & 2.0 & 8.2 & 27.3 & 11.8 & 3.088 & 23.1 & 41.5 \\
\hline 54 & N-31 (1.7 eq.) & $95 / 5$ & 500 & $\mathrm{I}$ & None & 20 & 0 & 3.6 & 15.0 & 55.3 & 22.7 & 3.442 & 23.9 & 39.3 \\
\hline 55 & $\mathrm{~N}-32$ (1.3 eq.) & $95 / 5$ & 500 & $\mathrm{I}$ & None & 20 & 0 & 6.1 & 24.0 & 83.4 & 35.2 & 3.229 & 24.5 & 40.9 \\
\hline 56 & $\mathrm{~N}-33$ (2.3 eq.) & $95 / 5$ & 500 & $\mathrm{I}$ & None & 20 & 0 & 2.1 & 8.1 & 27.1 & 11.7 & 3.094 & 22.9 & 39.5 \\
\hline 57 & $\mathrm{~N}-34$ (1.9 eq.) & $95 / 5$ & 500 & $\mathrm{I}$ & None & 20 & 0 & 2.9 & 12.6 & 46.6 & 19.1 & 3.473 & 24.6 & 41.5 \\
\hline 58 & $\mathrm{~N}-35$ (1.5 eq.) & $95 / 5$ & 500 & $\mathrm{I}$ & None & 20 & 0 & 5.0 & 20.7 & 72.3 & 30.2 & 3.251 & 24.7 & 41.5 \\
\hline 59 & $\mathrm{~N}-36$ (1.1 eq.) & $95 / 5$ & 500 & $\mathrm{I}$ & None & 20 & 0 & 7.6 & 27.7 & 92.3 & 40.0 & 3.062 & 28.8 & 48.3 \\
\hline 60 & $\mathrm{~N}-40$ & $50 / 50$ & 500 & $\mathrm{I}$ & Small & 50 & 0 & 42.2 & 115 & 196 & 137 & 1.340 & 92 & 155.4 \\
\hline 61 & $\mathrm{~N}-41$ & $95 / 5$ & 500 & $\mathrm{I}$ & Small & 50 & 0 & 11.5 & 74.4 & 139 & 75.0 & 1.715 & 46.8 & 86.5 \\
\hline 62 & $\mathrm{~N}-42$ & $95 / 5$ & 500 & $\mathrm{I}$ & Small & 20 & 0 & 3.7 & 26.5 & 99.9 & 43.3 & 3.628 & 31.4 & 56.1 \\
\hline 63 & $\mathrm{~N}-43$ & $50 / 50$ & 500 & $\mathrm{I}$ & Small & 20 & 0 & 3.6 & 19.5 & 96.0 & 36.7 & 4.740 & 35.2 & 72.5 \\
\hline
\end{tabular}


SI.3 Microscopy analysis of crystallization products with varying temperature and solvent composition

Polarized Light Microscopy images taken on slurries of acetone $/ \mathrm{H}_{2} \mathrm{O} 50 / 50$ and acetone $/ \mathrm{H}_{2} \mathrm{O} 95 / 5$ crystallized at different temperatures. In both cases particles are progressively larger from left to right, suggesting higher temperature favors larger particles. Comparing (a) and (b) series it is also evident that the rod-shape particles are generally thicker as water content increases. 
(a)
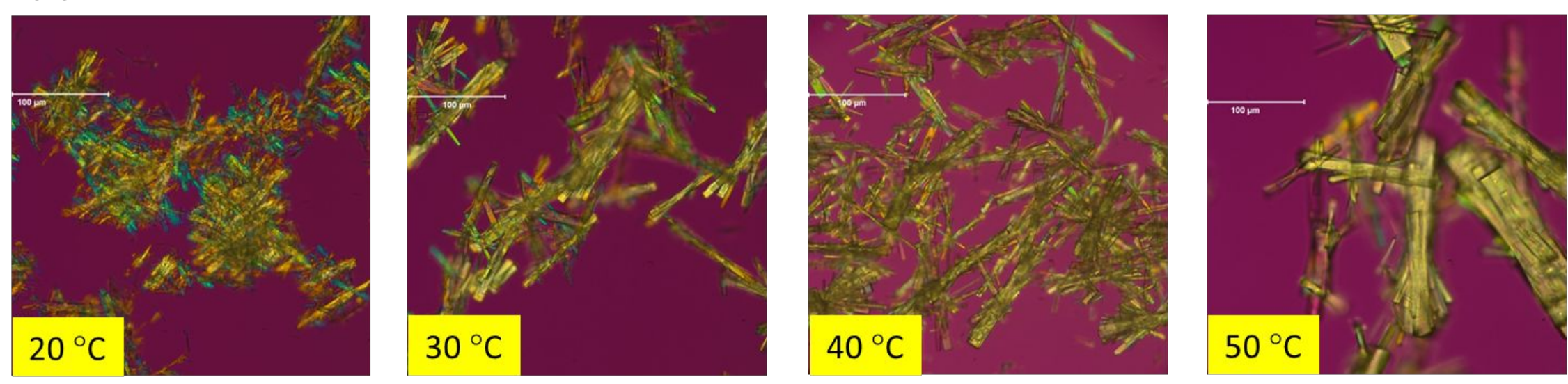

(b)


Figure SI.3.1. Microscopy images of crystallization in (1) acetone $/ \mathrm{H}_{2} \mathrm{O} 50 / 50$ series (b) acetone $/ \mathrm{H}_{2} \mathrm{O} 95 / 5$ series. 


\section{SI.4 Study on the aftereffect of temperature}

Two experiments were performed to evaluate the effect of temperature history after the crystallization event. The main purpose is to assess whether extended aging is a required operation of the process, and if PSD can be further manipulated through temperature cycling. In the first experiment, crystallization was conducted at $20{ }^{\circ} \mathrm{C}$ in water-deprived conditions (acetone $/ \mathrm{H}_{2} \mathrm{O}$ at 95/5). The batch was heated up to $50^{\circ} \mathrm{C}$ after the crystallization event. The Raman and temperature profile are shown in Figure SI.4.1. PSD sample taken before the heating ramp (M-46-02) and after $4 \mathrm{hr}$ aging at $50{ }^{\circ} \mathrm{C}$ (M-46-03) exhibited insignificant difference in morphology and PSD (Figure SI.4.2). This is direct evidence that the Maleate formation step is defining the particle size of the process. Subsequent treatment to dissolve small particles and grow larger particles via aging and temperature cycling have no effect on API particle size, likely due to insignificant solubility difference between 20 and $50^{\circ} \mathrm{C}$.



Figure SI.4.1. Raman and temperature profiles for experiment M-46 to assess aftereffect of temperature on PSD for acetone $/ \mathrm{H}_{2} \mathrm{O} 95 / 5$ crystallization. 


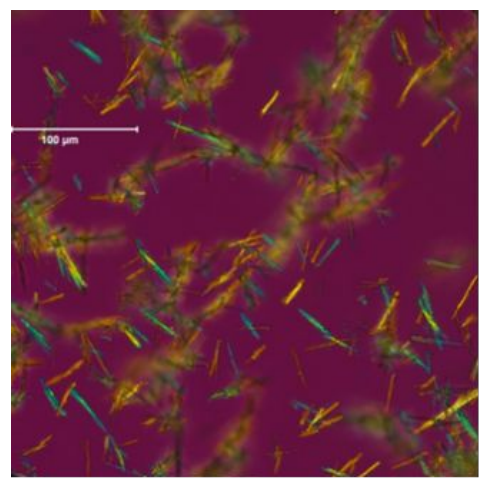

\begin{tabular}{|c|c|c|}
\hline $\begin{array}{c}\text { Before } \\
\text { temp } \\
\text { cycling }\end{array}$ & $\begin{array}{c}\text { Particle } \\
\text { size (um) }\end{array}$ & $\begin{array}{c}\text { After } \\
\text { temp } \\
\text { cycling }\end{array}$ \\
\hline 2.4 & $\mathrm{D}_{10}$ & 2.6 \\
\hline 10.3 & $\mathrm{D}_{50}$ & 10.6 \\
\hline 34.3 & $\mathrm{D}_{90}$ & 30.8 \\
\hline 14.8 & $\mathrm{D}[4,3]$ & 14 \\
\hline
\end{tabular}

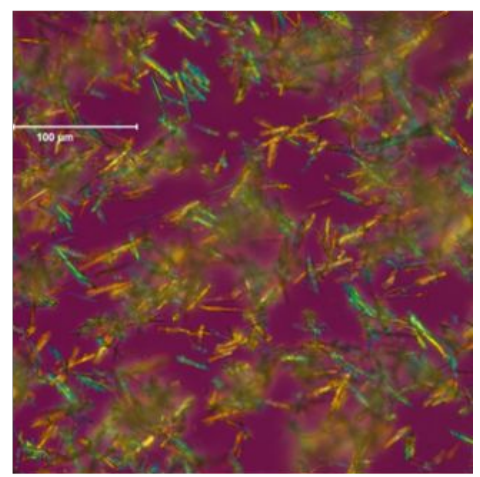

Figure SI.4.2. Microscopy and PSD results of experiment M-46 to assess aftereffect of temperature for acetone $/ \mathrm{H}_{2} \mathrm{O} 95 / 5$ crystallization.

A second experiment to verify the temperature aftereffect was conducted in water-rich compositions (acetone $/ \mathrm{H}_{2} \mathrm{O} 50 / 50$ ). The Raman and temperature profiles are shown in Figure SI.4.3. Similar to experiment (M-46), there was negligible difference in morphology and PSD before and after the temperature treatment (Figure SI.4.4). This result further confirms the insignificant effect of temperature after the Maleate formation.

A direct implication of this study is that extended aging of the crystallization slurry, a typical practice in pharmaceutical manufacturing, is not necessary from the form conversion perspective or from the PSD perspective. Any operation to manipulate API PSD has to be executed before or during the Maleate formation. This conclusion sets the tone for the crystallization studies in subsequent sections. 


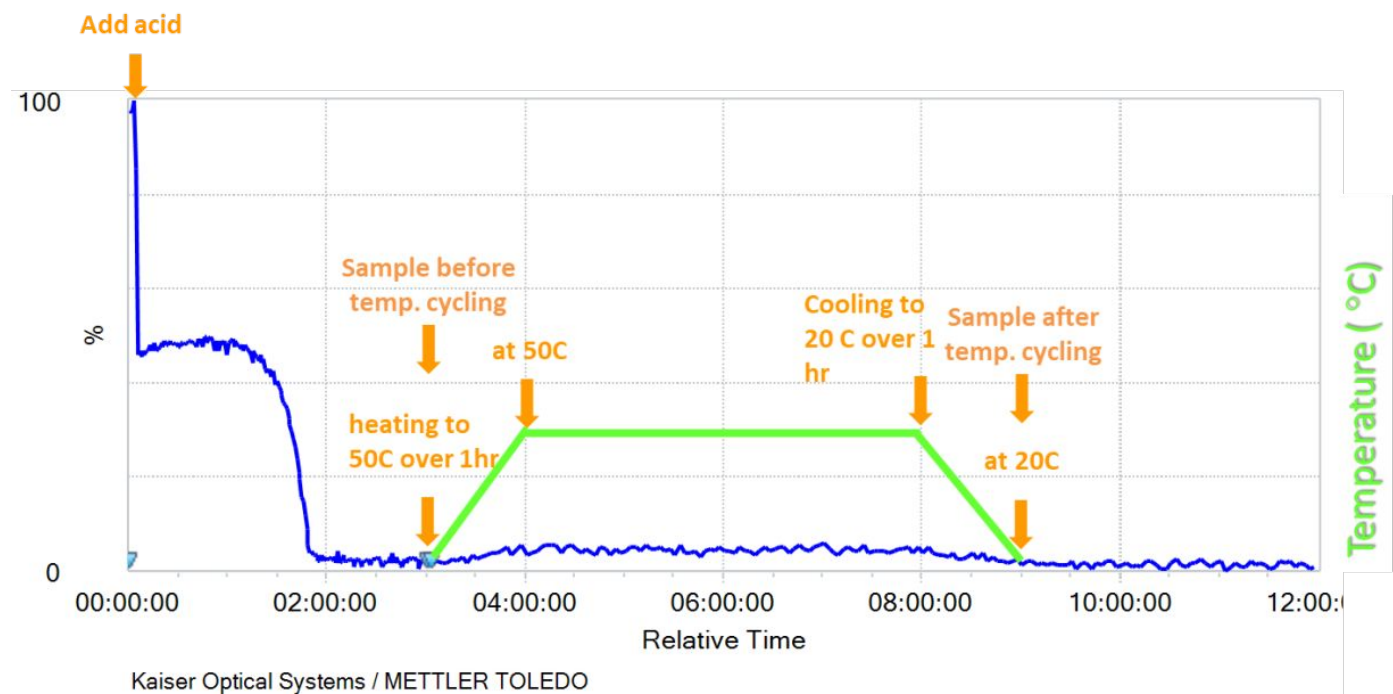

Figure SI.4.3. Raman and temperature profiles for experiment M-48 to assess aftereffect of temperature on PSD for acetone $/ \mathrm{H}_{2} \mathrm{O} 50 / 50$ crystallization.



\begin{tabular}{|c|c|c|}
\hline $\begin{array}{c}\text { Before } \\
\text { temp } \\
\text { cycling }\end{array}$ & $\begin{array}{c}\text { Particle } \\
\text { size (um) }\end{array}$ & $\begin{array}{c}\text { After } \\
\text { temp } \\
\text { cycling }\end{array}$ \\
\hline 2.7 & $\mathrm{D}_{10}$ & 3.9 \\
\hline 14.9 & $\mathrm{D}_{50}$ & 17.7 \\
\hline 54.0 & $\mathrm{D}_{90}$ & 46.0 \\
\hline 22.1 & $\mathrm{D}[4,3]$ & 22.0 \\
\hline
\end{tabular}

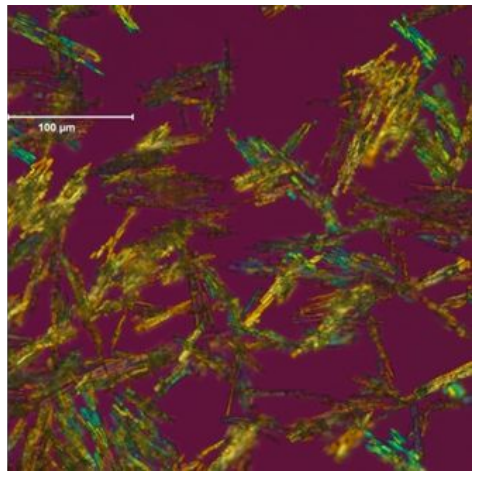

Figure SI.4.4. Microscopy and PSD results of experiment M-48 to assess aftereffect of temperature for acetone $/ \mathrm{H}_{2} \mathrm{O} 50 / 50$ crystallization.

\section{SI.5 Statistical analysis of crystallization data in Table SI.2}

The $\mathrm{R}^{2}$ value of 0.97 and the $\mathrm{R}^{2}$-adjusted value of 0.97 reflect the agreement between the model and the observed responses (Figure SI.5.1). The $p$-value of the ANOVA $F$-test supports the statistical significance of the model for the D90 response (Figure SI.5.2). 


\begin{tabular}{|lr|}
\hline Summary of Fit & \\
\hline RSquare & 0.972666 \\
\hline RSquare Adj & 0.967954 \\
Root Mean Square Error & 11.57579 \\
\hline Mean of Response & 98.10286 \\
\hline Observations (or Sum Wgts) & 35 \\
\hline
\end{tabular}

Figure SI.5.1. Summary of Fit

\begin{tabular}{|l|r|r|r|r|}
\hline \multicolumn{5}{|l|}{ Analysis of Variance } \\
\\
Source & DF & $\begin{array}{r}\text { Sum of } \\
\text { Squares }\end{array}$ & Mean Square & F Ratio \\
Model & 5 & 138282.10 & 27656.4 & 206.3928 \\
Error & 29 & 3885.97 & 134.0 & Prob > F \\
C. Total & 34 & 142168.07 & & $<.0001^{*}$
\end{tabular}

Figure SI.5.2. Analysis of Variance

The terms in the model for the D90 response are summarized in Figure SI.5.3 and Figure SI.5.4. Based on the analysis, solvent composition has the largest impact on D90. This is reflected in the large, statistically significant effect of the main and quadratic term for \%acetone. Temperature has the largest positive effect on D90. The quadratic term for temperature was not found to be statistically significant in this analysis. The interactions are depicted graphically in Figure SI.5.5.

\begin{tabular}{|c|c|c|c|c|c|}
\hline \multicolumn{6}{|l|}{ Effect Tests } \\
\hline Source & Nparm & DF & $\begin{array}{l}\text { Sum of } \\
\text { Squares }\end{array}$ & F Ratio & Prob $>F$ \\
\hline \%Acetone & 1 & 1 & 50808.161 & 379.1683 & $<.0001^{*}$ \\
\hline Temp (deg C) & 1 & 1 & 37204.733 & 277.6494 & $<.0001^{*}$ \\
\hline \%Acetone*\%Acetone & 1 & 1 & 24194.613 & 180.5582 & $<.0001^{*}$ \\
\hline \%Acetone*Temp (deg C) & 1 & 1 & 7338.673 & 54.7666 & $<.0001^{*}$ \\
\hline Temp $(\operatorname{deg} C)^{*}$ Temp $(\operatorname{deg} C)$ & 1 & 1 & 712.082 & 5.3141 & $0.0285^{*}$ \\
\hline
\end{tabular}

Figure SI.5.3. Effect tests

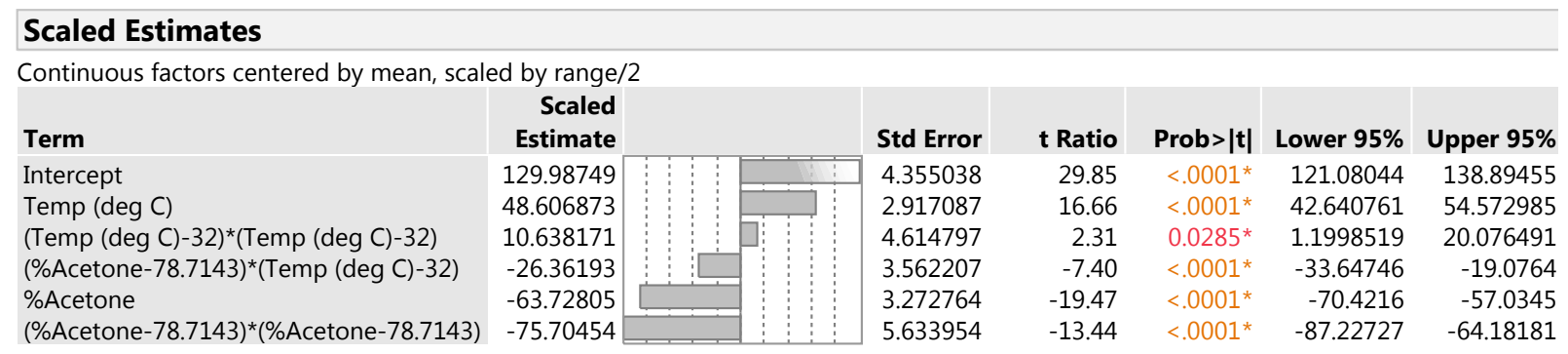

Figure SI.5.4. Scaled estimates 


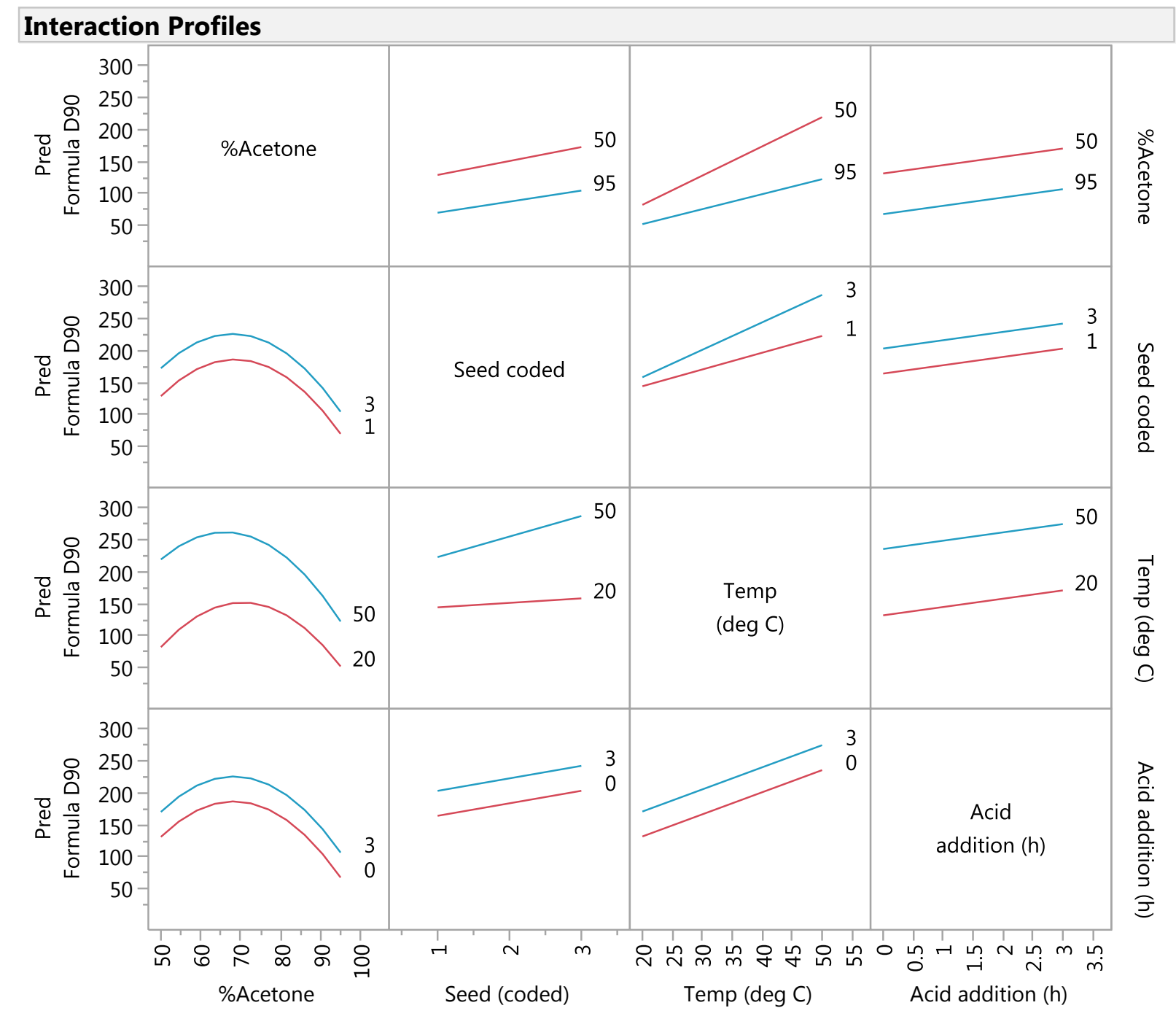

Figure SI.5.5. Interaction profiles 